\title{
Thermal phase diagrams of columnar liquid crystals
}

\author{
G. Lamoureux, A. Caillé and D. Sénéchal \\ Département de physique and Centre de recherche en physique du solide, \\ Université de Sherbrooke, Sherbrooke, Québec, Canada J1K 2R1.
}

\begin{abstract}
In order to understand the possible sequence of transitions from the disordered columnar phase to the helical phase in hexa(hexylthio)triphenylene (HHTT), we study a three-dimensional planar model with octupolar interactions inscribed on a triangular lattice of columns. We obtain thermal phase diagrams using a mean-field approximation and Monte Carlo simulations. These two approaches give similar results, namely, in the quasi one-dimensional regime, as the temperature is lowered, the columns order with a linear polarization, whereas helical phases develop at lower temperatures. The helicity patterns of the helical phases are determined by the exact nature of the frustration in the system, itself related to the octupolar nature of the molecules.
\end{abstract}

\section{INTRODUCTION}

The study of phase transitions in columnar liquid crystals [1,2] presents a fundamental interest in that these materials combine, aside from the vast phenomenology of soft matter, many features at the origin of important phenomena of the solid state, namely a relatively strong elastic anisotropy in the direction of the columns, a geometrical frustration of the intermolecular interaction coming from the triangular nature of the lattice of columns, and finally discoid molecules with nontrivial point-group symmetry. The present study is based on hexa(hexylthio)triphenylene (HHTT), a compound formed of a rigid core of aromatic cycles, giving a discoid shape to the molecule, and of six flexible hydrocarbon chains, responsible for its characteristic thermotropic character. HHTT is the only molecule from the triphenylene derivatives to show two distinct columnar phases. Indeed, as the temperature decreases, the sequence of phases is the following: $I$, an isotropic liquid; $D_{h d}$, a disordered columnar phase; $H$, a helically ordered columnar phase and finally $K$, a monoclinic crystal. These phases were identified by X-rays measurements on powders [3] and freely suspended strands [4] of HHTT.

These X-rays results are best interpreted by concluding that the $D_{h d}$ phase of HHTT $\left(70^{\circ} \mathrm{C}<T<93^{\circ} \mathrm{C}\right)$ has long-range positional order in the plane perpendicular to the columns: columns are located on a triangular lattice. Within a column, short range (liquid) positional order is realized. The columns slide freely one against the other. The $H$ phase $\left(62^{\circ} \mathrm{C}<T<70^{\circ} \mathrm{C}\right)$ has in-column long-range positional and orientational helical order, or quasi long-range order, as proposed in [7]. In this last phase, two neighboring molecules in a single column are separated on the average by a distance $d_{\|}=3.6 \AA$ and rotated from each other by an angle $\alpha \approx 45^{\circ}$, constant on the whole temperature interval of the phase. In order to reduce the frustration associated with the triangular geometry of the lattice, the lattice reorganizes itself in a superlattice $\sqrt{3} \times \sqrt{3} R 30^{\circ}$ : one third of the columns have a vertical offset of half the inter-molecular distance $\left(d_{\|} / 2\right)$. The displaced columns have an opposite helicity: $\alpha \approx-45^{\circ}$, instead of $+45^{\circ}$ for the undisplaced columns. If it were not for the very high value of the mean square displacement of the molecules in the direction of the columns, the $H$ phase would seem very similar to a crystalline phase. In that sense, the exact mechanism of the $D_{h d} \leftrightarrow H$ transition is still an open question. In an effort towards elucitating the mechanism, we study the orientational ordering assuming from the start that the HHTT molecules already occupy well defined positions.

We study the thermal phase diagram of a model Hamiltonian by means of a Landau free energy functional in a mean-field approximation and of Monte Carlo simulations on finite size lattice. Previous work has been done on the ground state of a related model [8] and on thermal phase diagrams for a two-dimensional model of uniform columns [9, 10]. Our analysis confirms that, as previously seen at $T=0$, the octupolar $G$ coupling [11] is determinant for obtaining the helicity configuration of the columns at any temperature. It also shows that a diversity of phases survives at $T \neq 0$. For weak transverse couplings, the model produces the expected lowtemperature helical phases, but also suggests that some linearly polarized phases could exist at higher temperatures.

In the following section, the model Hamiltonian is presented with an emphasis on the inter-columnar intermolecular interactions. In Sec. III, using mean-field approaches, the locus of points for the second order phase transitions from the disordered phase to an ordred phase is obtained. It is followed by a determination of the thermal phase diagrams and characterisation of the different phases in terms of the helicity pattern and relative phases. In Sec. IV, the thermal phase diagrams are obtained using Monte Carlo simulations on finite size systems in conjunction with the spiraling algorithm. Finally, Sec. V discusses the results and arrives at general conclusions. 


\section{MODEL HAMILTONIAN}

As indicated it the introduction, the main purpose of our calculation is to elucidate the role played by the angular degrees of freedom. Even though this may be questionable formally, we will freeze the positional degrees of freedom to render the problem tractable. Despite the fact that the exact positional order in the columns is not known, we then assume that the molecules lie on a three-dimensional triangular lattice of ordered columns (with one third of the columns being displaced). This simplification is certainly valid in the $H$ phase, even if only quasi long-range positional order existed since order would then be maintained over many inter-molecular distances. Accordingly, we will use $(i, j)$ site indices to identify the unit cell of the three columns of the twodimensional $\sqrt{3} \times \sqrt{3} R 30^{\circ}$ superlattice of columns and $\mu$ to identify the column: $\mu=1$ and 2 label the undisplaced columns and $\mu=3$ labels the columns offset by $d_{\|} / 2$. Finally, an index $k$ identifies the sites a molecule occupies in the $(\mu, i, j)$ column. We formally write $(i, j, k)$ as $m$, an index labelling a plaquette of three molecules. a)

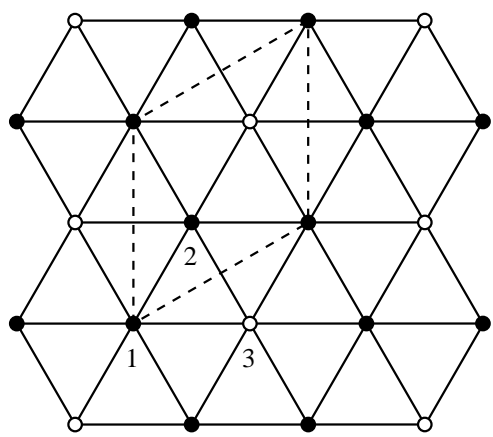

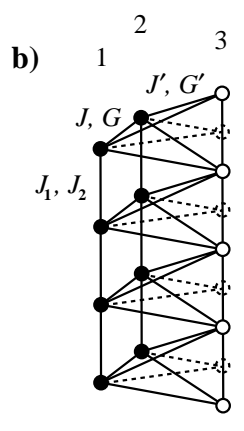

FIG. 1. a) Two-dimensional triangular lattice of columns. Empty dots represent displaced columns $(\mu=3)$ and filled dots, undisplaced columns $(\mu=1$ and 2$)$. Dashed lines represent the elementary cell of the super-lattice. b) Three-dimensional illustration of the couplings for three columns. Dotted lines represent fictive sites and interactions for an undisplaced column 3 .

Within the framework of this plastic state model, each molecule $(\mu, m)$ has a well-defined position $\mathbf{r}_{\mu m}$ and an orientation labelled $\theta_{\mu m}$, admitting from the beginning that its plane is perpendicular to the direction of the columns. An orientational disorder may mimick an effective $D_{h d}$ phase, which however would possess orientational and positional disorders along the columns. We did not explicitly consider the shape and flexibility of the tails, which would vary with the temperature. Recall, however, that it has been suggested [4,5] that the stiffening of the tails may be responsible for the $D_{h d} \rightarrow H$ transition. The above considerations are implicitly integrated out as weakly temperature-dependent renormalization effects of the inter-columnar interactions, allowing us to use, to a good approximation, an effective model with athermal values of the interaction parameters. The resulting thermal phase diagrams will represent a somewhat distorted version of the true temperature dependence.

The last thing to consider in the model is that the molecules are not exactly disk-like: they have a chirality associated with the alternate arrangement of the aliphatic tails. Indeed, conformational analysis on compounds similar to HHTT 12, 13] shows that this "propeller blade" configuration is the ground state of a single molecule and of two stacked molecules, one on top of the other.

\section{A. Intra-columnar interactions}

In their ground state, two stacked HHTT molecules minimize their conformation energy by allowing an angular shift $\alpha$ between the two molecules [12,13. An intrinsic chiral model represented by the Hamiltonian

$$
-J \cos 3\left(\theta_{k+1}-\theta_{k}-\alpha\right),
$$

would be appropriate to represent this situation since the HHTT molecules have $D_{3}$ symmetry. However there is no a priori selection between the right-handed or left-handed chirality. In order to allow for the two possible chiralities of each column (the sign of $\alpha$ ), we preferably use a next-nearest-neighbor model (see 14,15]), with competing interactions of the form:

$$
-J_{1} \cos 3\left(\theta_{k+1}-\theta_{k}\right)-J_{2} \cos 3\left(\theta_{k+2}-\theta_{k}\right),
$$

with $J_{1}>0$ and $J_{2}<0$. In the ground state, for $4\left|J_{2}\right| \geq\left|J_{1}\right|$, the molecules adopt a helical configuration with pitch $\alpha$, given by $\cos 3 \alpha=-J_{1} / 4 J_{2}$. Otherwise, the intrinsinc helicity of the columns is zero. The $J_{1} / J_{2}$ ratio determines the magnitude of $\alpha$, but allows opposite helicities for different columns or even helicity reversals within a column, separating helicity domains.

For an isolated molecule of HHTT at finite temperature, it is unclear that chirality is a well defined property: conformational analysis [12] shows that the energy barrier between opposite chirality configurations is comparable to the thermal energy in the $H$ and $D_{h d}$ phases. Thus, instead of using an additional Ising variable on each site to represent the chirality of the molecule, we represent the chirality as the result of a collective behavior in the effective model (2), submitted to inter-columnar interactions: every molecule in a particular helicity domain has the same chirality, related to the sign of the helicity. The inter-columnar interactions are essential to stabilize helicity domains at finite temperature.

\section{B. Inter-columnar interactions}

Given the approximation that each molecule is fully described by its orientation $\theta_{\mu m}$, we may write its mass 
density as a multipole expansion [11, 16]. Because of the $D_{3}$ point symmetry of the molecule, the first nonzero moment is the octupolar moment, which may be represented by $Q_{k l m}$, a rank-three tensor $(k, l, m=x, y)$. The only interactions that are bilinear in $Q$ as well as invariant with respect to the symmetries of the hexagonal lattice have a $\cos 3\left(\theta-\theta^{\prime}\right)$ or $\cos 3\left(\theta+\theta^{\prime}\right)$ form [11]. The intercolumnar interaction is then approximated to be

$$
-J \cos 3\left(\theta_{\mu i j}-\theta_{\mu^{\prime} i^{\prime} j^{\prime}}\right)-G \cos 3\left(\theta_{\mu i j}+\theta_{\mu^{\prime} i^{\prime} j^{\prime}}\right) .
$$

The first term is invariant under continuous rotations and would be present even if the molecules had lower symmetry multipole moments. However, the second term is specific to the octupolar character of the molecules and has only discrete rotational symmetries.

To extract the $D_{3}$ symmetry of the molecules, we replace the real orientations $\theta_{\mu m}$ by angular variables $\phi_{\mu m}=3 \theta_{\mu m}$. The complete hamiltonian of the system then reads:

$$
\begin{aligned}
& H=-\sum_{\mu \nu} \sum_{m n} {\left[J_{\mu m, \nu n} \cos \left(\phi_{\mu m}-\phi_{\nu n}\right)\right.} \\
&\left.+G_{\mu m, \nu n} \cos \left(\phi_{\mu m}+\phi_{\nu n}\right)\right] .
\end{aligned}
$$

$J_{\mu m, \nu n}$ contains the intra-columnar interactions: each site is coupled to its first and second intra-column nearest neighbors by $J_{1}$ and $J_{2}$ as in Eq. (2). The intercolumnar couplings are embedded both in $J_{\mu m, \nu n}$ and $G_{\mu m, \nu n}$. Each $\mu=1$ site interacts with three $\mu=2$ and six $\mu=3$ neighbors, three upwards and three downwards. The inter-columnar couplings have different values: $J$ and $G$ for in-plane molecules (1-2 bonds) and $J^{\prime}$ and $G^{\prime}$ for out-of-plane molecules (1-3 and 2-3 bonds) (see Fig. 1 b). Nevertheless, it is physically justified [9] to suppose that $J^{\prime} \approx J$ and $G^{\prime} \approx G$, and for simplicity, we assume $J^{\prime}=J$ and $G^{\prime}=G$. $J_{1}$ is positive and taken to be unity (it sets the energy scale).

With the notations

$$
c_{\mu m}=\cos \left(\phi_{\mu m}\right) \quad \text { and } \quad s_{\mu m}=\sin \left(\phi_{\mu m}\right),
$$

we may re-write the hamiltonian (仼) as

$$
H=-\sum_{\mu \nu} \sum_{m n}\left[J_{\mu m, \nu n}^{c} c_{\mu m} c_{\nu n}+J_{\mu m, \nu n}^{s} s_{\mu m} s_{\nu n}\right],
$$

where $J_{\mu m, \nu n}^{c}=J_{\mu m, \nu n}+G_{\mu m, \nu n}$ and $J_{\mu m, \nu n}^{s}=J_{\mu m, \nu n}-$ $G_{\mu m, \nu n}$. The reader should note that the $G \leftrightarrow-G$ transformation interchanges the $c$ and $s$ variables and is equivalent to a rotation $\phi_{\mu m} \leftrightarrow \phi_{\mu m}+\frac{\pi}{2}$ of the molecules.

\section{MEAN-FIELD CALCULATION}

We use a six-component variable $S_{i m}=\left(c_{1 m}, c_{2 m}, c_{3 m}, s_{1 m}, s_{2 m}, s_{3 m}\right)$. In a Fourier representation, the mean-field is

$$
h_{i}(\mathbf{q})=\sum_{j} J_{i j}(\mathbf{q})\left\langle S_{j}(\mathbf{q})\right\rangle,
$$

where $J_{i j}(\mathbf{q})$ is a $6 \times 6$ block diagonal matrix constructed from the $J^{c}$ and $J^{s}$ couplings of Eq. (6):

$$
J(\mathbf{q})=\left(\begin{array}{cc}
J^{c}(\mathbf{q}) & 0 \\
0 & J^{s}(\mathbf{q})
\end{array}\right) .
$$

Because there are three columns in the unit cell, the mean-field transverse components of $\mathbf{q}$ are zero, as we have verified through a detailed calculation. From now on, without any ambiguity, we replace $\mathbf{q}$ by $q$, its $z$ component. We also take the intra-column distance between two molecules $d_{\|}=1$. The $J^{c}$ and $J^{s}$ matrices are then

$$
J^{c, s}(q)=\left(\begin{array}{ccc}
J_{\|}(q) & J_{12}^{c, s} & J_{31}^{c, s}(q) \\
J_{12}^{c, s} & J_{\|}(q) & J_{23}^{c, s}(q) \\
J_{31}^{c, s}(q) & J_{23}^{c, s}(q) & J_{\|}(q)
\end{array}\right),
$$

with $J_{\|}(q)=\cos q+J_{2} \cos 2 q, J_{12}^{c}=\frac{3}{2}(J+G), J_{23}^{c}(q)=$ $J_{31}^{c}(q)=3(J+G) \cos \frac{1}{2} q, J_{12}^{s}=\frac{3}{2}(J-G)$ and $J_{23}^{s}(q)=$ $J_{31}^{s}(q)=3(J-G) \cos \frac{1}{2} q$. The displacement of the $\mu=3$ columns changes the coordination number from 6 to 3 and adds a $\cos \frac{1}{2} q$ factor.

\section{A. Second-order phase transition temperature $T_{c}$}

The order-disorder continuous phase transitions are related to the divergence of the "paramagnetic" susceptibility $\chi$, which in turn is related to the single-site susceptibility $\chi_{0}=1 / 2 T$, with $k_{B}=1$, by the standard RPA relation:

$$
\chi(q)=\chi_{0}\left[\mathbb{1}-\chi_{0} J(q)\right]^{-1} .
$$

The $6 \times 6$ matrix between brackets is non-invertible when at least one of its eigenvalues is zero. As the temperature is lowered, the transition occurs for some $q_{c}$ maximizing one of the six eigenvalues of $J(q)$. The corresponding eigenvector identifies the configuration involved in the transition. The eigenvalue itself is twice the critical temperature $T_{c}$.

$J(q)$ is block-diagonal, and the 6th-order caracteristic equation reduces to the two cubic equations given by

$$
\operatorname{det}\left(J^{c, s}(q)-j^{c, s} \mathbb{1}\right)=0 .
$$

The eigenvalues are

$$
j_{1}^{c, s}(q)=J_{\|}(q)-J_{12}^{c, s},
$$

$$
j_{2}^{c, s}(q)=J_{\|}(q)+\frac{1}{2}\left[J_{12}^{c, s}-\sqrt{J_{12}^{c, s}{ }^{2}+8 J_{23}^{c, s}(q)^{2}}\right],
$$

$$
j_{3}^{c, s}(q)=J_{\|}(q)+\frac{1}{2}\left[J_{12}^{c, s}+\sqrt{J_{12}^{c, s 2}+8 J_{23}^{c, s}(q)^{2}}\right] .
$$


The eigenvectors are of the form

$$
\begin{gathered}
v_{1}^{c, s}(q)=(1,-1,0), \\
v_{2}^{c, s}(q)=\left(j_{2}^{c, s}-J_{\|}(q), j_{2}^{c, s}-J_{\|}(q), 2 J_{23}^{c, s}(q)\right), \\
v_{3}^{c, s}(q)=\left(j_{3}^{c, s}-J_{\|}(q), j_{3}^{c, s}-J_{\|}(q), 2 J_{23}^{c, s}(q)\right) .
\end{gathered}
$$

For each parameter set $\left(J, G, J_{2}\right)$, we numerically find which of the six eigenvalues is maximal and the corresponding $q_{c}$. It turns out that the only two eigenvalues to be maximum are $j_{3}^{c}$ and $j_{3}^{s}$. The critical temperature is thus the maximum of the following two temperatures:

$$
\begin{aligned}
T_{c}^{c, s}= & \max \left(\frac{1}{2} j_{3}^{c, s}\right) \\
= & \frac{1}{2}\left(\cos q_{c}+J_{2} \cos 2 q_{c}\right)+\frac{3}{8}(J \pm G) \\
& +\frac{3}{8}|J \pm G| \sqrt{1+32 \cos ^{2} \frac{1}{2} q_{c} .}
\end{aligned}
$$

If $T_{c}^{c}>T_{c}^{s}$ (resp. $T_{c}^{c}<T_{c}^{s}$ ), only the cosine (resp. sine) components are involved in the transition. The boundary between the cosine and sine transitions is defined by $T_{c}^{c}=T_{c}^{s}$. Noticing the $(a, a, b)$ structure of the $v_{3}^{c}$ and $v_{3}^{s}$ vectors, we conclude that columns 1 and 2 play similar roles, while column 3 has a distinct behavior.

In the $J-G$ plane, and for a particular value of $J_{2}$, we identify four regions corresponding roughly to the four quadrants, two of them being shown in Fig. 2. The line $G=0$, where the cosine and sine components are equivalent, is an obvious boundary. The curve

$$
|G|=-g\left(J, J_{2}\right) J
$$

on which $T_{c}^{c}=T_{c}^{s}$, follows from a mechanism similar to the spin-flop mechanism of magnetism. For $|J|$ and $|G|$ sufficiently high relative to $\left|J_{2}\right|, q_{c}$ vanishes, that is, the transverse couplings have "untwisted" the columns. The equation $T_{c}^{c}\left(q_{c}=0\right)=T_{c}^{s}\left(q_{c}=0\right)$ then gives a relation between $J$ and $G$ :

$$
(J+G)+|J+G| \sqrt{33}=(J-G)+|J-G| \sqrt{33} .
$$

The solutions are $G=0$ and $|G|=-\sqrt{33} J$. Thus, in this limit of $q_{c}=0, g=\sqrt{33} \approx 5.74$, independently of $J$ or $J_{2}$. At $q_{c}=0$, the columns behave like a single vector flipping under the anisotropic effect of $G$. For smaller values of $|J|$ and $|G|, q_{c} \neq 0$ and $g$ decreases, the same mechanism however remains.

In the "cosine" regions, the helicity at the transition is a function $q_{c}=q_{c}\left(J+G, J_{2}\right)$, because $J+G$ is the only combination of $J$ and $G$ appearing in $j_{3}^{c}$. In the "sine" regions, we have $q_{c}=q_{c}\left(J-G, J_{2}\right)$ for the same reason. For $J_{2}>-\frac{1}{4}, q_{c}=0$, that is, each column stabilizes a "ferromagnetic" order 14, 15]. For $J_{2}<-\frac{1}{4}$, there is a region of the $J-G$ plane where $q_{c} \neq 0$, but $q_{c}=0$ for $|J|$ and $|G|$ high enough. The boundary is determined by the competition between $\left|J_{2}\right|$ and the transverse couplings, respectively inducing a modulation in the columns, and favoring $q_{c}=0$.

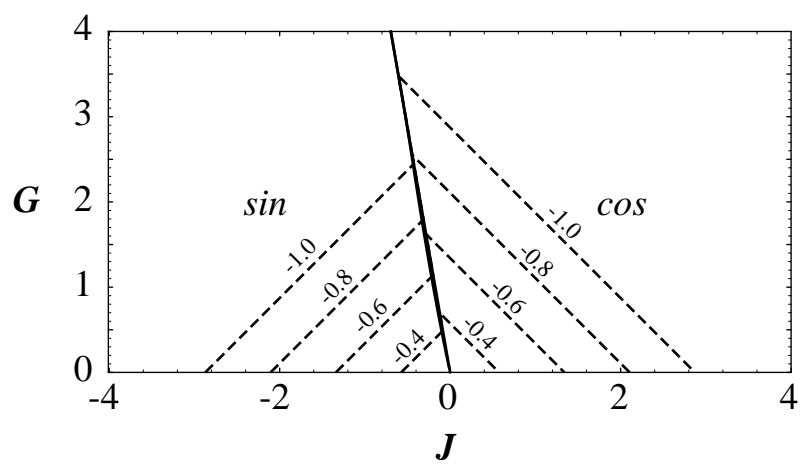

FIG. 2. Phase diagrams at the critical temperature for $J_{2}=-1.0,-0.8,-0.6$ and -0.4 . The full lines, all superimposed with the precision used, are the boundaries between $\cos$ and $\sin$ phases and the dashed lines, the boundaries between $q_{c}=0$ and $q_{c} \neq 0$ phases. For $G<0$, the boundaries are a mirror image of the above with cos and sin phases being interverted.

\section{B. Thermal phase diagrams}

In order to investigate finite-temperature effects near the order-disorder transition, to better specify the nature of the phases, and to rule out the possibility of higher temperature first-order transitions, we set up a Landau theory from the microscopic model. We follow in essence the method proposed by Bak and von Boehm 17].

\section{Free energy functional expansion}

For commodity, we divide the Landau free energy functional $F$ into two parts: $F_{T}$ and $F_{J}$. In reciprocal space, to fourth order, we find that

$$
\begin{aligned}
F_{T}= & T \sum_{\mu}\left\{\sum_{q_{1}}\left[c_{\mu}\left(q_{1}\right) c_{\mu}\left(-q_{1}\right)+s_{\mu}\left(q_{1}\right) s_{\mu}\left(-q_{1}\right)\right]\right. \\
& +\frac{1}{4} \sum_{q_{1} q_{2} q_{3}}\left[c_{\mu}\left(q_{1}\right) c_{\mu}\left(q_{2}\right) c_{\mu}\left(q_{3}\right) c_{\mu}\left(-q_{1}-q_{2}-q_{3}\right)\right. \\
& +2 c_{\mu}\left(q_{1}\right) c_{\mu}\left(q_{2}\right) s_{\mu}\left(q_{3}\right) s_{\mu}\left(-q_{1}-q_{2}-q_{3}\right) \\
& \left.\left.+s_{\mu}\left(q_{1}\right) s_{\mu}\left(q_{2}\right) s_{\mu}\left(q_{3}\right) s_{\mu}\left(-q_{1}-q_{2}-q_{3}\right)\right]\right\}
\end{aligned}
$$

with $T=1 / \beta . \quad c_{\mu}(\tilde{q})$ and $s_{\mu}(\tilde{q})$ are the Fourier transforms of the mean values $\left\langle c_{\mu m}\right\rangle$ and $\left\langle s_{\mu m}\right\rangle$. This limited power expansion is numerically close to (less than $1 \%$ difference) the exact value up to $\sqrt{\left\langle c_{\mu m}\right\rangle^{2}+\left\langle s_{\mu m}\right\rangle^{2}} \approx$ 0.5. A 6th-order development is $1 \%$ accurate up to $\sqrt{\left\langle c_{\mu m}\right\rangle^{2}+\left\langle s_{\mu m}\right\rangle^{2}} \approx 0.65$. For every mean value under this limit of validity, a negligible number of ficti- 
tious spins with modulus higher than 1 contributes to the statistics. We also find that

$$
\begin{aligned}
F_{J}=-\sum_{\mu \nu} \sum_{q_{1}} & {\left[J_{\mu \nu}^{c}\left(q_{1}\right) c_{\mu}\left(q_{1}\right) c_{\nu}\left(-q_{1}\right)\right.} \\
& \left.+J_{\mu \nu}^{s}\left(q_{1}\right) s_{\mu}\left(q_{1}\right) s_{\nu}\left(-q_{1}\right)\right] .
\end{aligned}
$$

These expressions represent the free energy functional for a group of three columns. The $q$ summations have been truncated of all the umklapp terms. These umklapp terms would have pinned the modulation to commensurate values. By ignoring them, we allow incommensurate phases to occupy the entire parameter space, leaving a space of measure zero to commensurate phases. In real systems a devil's staircase [17] is expected instead of the continuous $q$ profile.

\section{Order parameters}

We then assume that, near the transition, $c_{\mu}(\tilde{q})=0$ and $s_{\mu}(\tilde{q})=0(\forall \mu)$ except for $\tilde{q}= \pm q$. In other words, we concentrate on the first harmonic to appear in the modulated phases. This is valid at the transition but it is not excluded that higher harmonics may appear at lower temperatures, as secondary order parameters. $c_{\mu}(q)$ and $s_{\mu}(q)$ are the $x$ and $y$ components of three polarization vectors

$$
\mathbf{S}_{\mu}(q)=c_{\mu}(q) \hat{\mathbf{x}}+s_{\mu}(q) \hat{\mathbf{y}} .
$$

These are complex quantities that may be expressed as

$$
c_{\mu}=\left|c_{\mu}\right| e^{i \varphi_{\mu}^{c}} \quad \text { and } \quad s_{\mu}=\left|s_{\mu}\right| e^{i \varphi_{\mu}^{s}} .
$$

This choice of variables allows for any elliptical polarization and relative global phase for each column. To simplify notation, we replace $\left|c_{\mu}\right|$ by $c_{\mu}$ and $\left|s_{\mu}\right|$ by $s_{\mu}$ and, to avoid any ambiguity, we make no use of the complex $c_{\mu}$ and $s_{\mu}$ anymore. In real space,

$$
\begin{aligned}
\left\langle\mathbf{S}_{\mu k}\right\rangle & =\frac{1}{2}\left[\mathbf{S}_{\mu}(q) e^{i q z_{k}}+\mathbf{S}_{\mu}^{*}(q) e^{-i q z_{k}}\right] \\
& =c_{\mu} \cos \left(q z_{k}+\varphi_{\mu}^{c}\right) \hat{\mathbf{x}}+s_{\mu} \cos \left(q z_{k}+\varphi_{\mu}^{s}\right) \hat{\mathbf{y}},
\end{aligned}
$$

with $z_{k}=k+\frac{1}{2} \delta_{\mu 3}$, so that $\varphi_{\mu}^{c, s}$ are the global phases of the different columns $\mu$ at the $z=0$ level.

The function to minimize is then $F=F_{T}+F_{J}$, with

$$
\begin{aligned}
F_{T}=T \sum_{\mu}\{ & 2\left(c_{\mu}^{2}+s_{\mu}^{2}\right)+\frac{1}{4}\left[6\left(c_{\mu}^{4}+s_{\mu}^{4}\right)\right. \\
& \left.\left.+4\left[2+\cos 2\left(\varphi_{\mu}^{s}-\varphi_{\mu}^{c}\right)\right] c_{\mu}^{2} s_{\mu}^{2}\right]\right\}
\end{aligned}
$$

and

$$
\begin{aligned}
F_{J}=-\sum_{\mu \nu}[ & J_{\mu \nu}^{c} c_{\mu} c_{\nu} \cos \left(\varphi_{\nu}^{c}-\varphi_{\mu}^{c}\right) \\
& \left.+J_{\mu \nu}^{s} s_{\mu} s_{\nu} \cos \left(\varphi_{\nu}^{s}-\varphi_{\mu}^{s}\right)\right],
\end{aligned}
$$

with couplings as previously defined. $F$ is a function of $c_{\mu}, s_{\mu}, \varphi_{\mu}^{c, s}$ and $q$ that, at first, is numerically minimized. The reader should note that the permutation $1 \leftrightarrow 2$ in the indices leaves $F$ unchanged, which reflects the equivalence of columns 1 and 2 .

\section{Helicity patterns}

We numerically observe simple relationships between the phases $\varphi_{\mu}^{c}$ and $\varphi_{\mu}^{s}$. These, in turn, lead to a simplified expression for the free energy functional. For $G=0$, the cosine and sine components are equivalent and $s_{\mu}=c_{\mu}$. By numerically minimizing $F$, we obtain

$$
\varphi_{\mu}^{s}-\varphi_{\mu}^{c}= \pm \frac{\pi}{2},
$$

so that the modulation appearing in the columns is circularly polarized, with a helicity given by the sign on the right-hand side. This sign $\left(\sigma_{\mu}= \pm\right)$ may differ from one $\mu$ value to another. We denote $\left(\sigma_{1}, \sigma_{2}, \sigma_{3}\right)$ the helicity configuration of the three sublattices of columns. For $G=0$, the only allowed helicity configuration is $(+++)$ (or equivalently $(---)$ ). Depending on the sign of $J$, the relative global phases of each component from one column to another are 0 or $\pm \pi$. For $J>0$, the columns adopt a "ferromagnetic-like" arrangement:

$$
\varphi_{1}^{c, s}=\varphi_{2}^{c, s}=\varphi_{3}^{c, s},
$$

and for $J<0$, the triangular geometry imposes a colinear "antiphase":

$$
\varphi_{1}^{c, s}=\varphi_{2}^{c, s}=\varphi_{3}^{c, s} \pm \pi .
$$

Instead of the ordinary " $120^{\circ}$ state" for an evenly frustrated system, our system concentrates the frustration in the 1-2 bond, which has a lower coordination number than 1-3 and 2-3 bonds. In contrast to the results obtained at $T=0$ in [\$], where every column was forced to adopt the same amplitude and a deformed " $120^{\circ}$ state" was achieved, we obtained here a fully colinear antiphase.

This fundamental difference arises from the freedom of the above model to adopt different amplitudes of modulation for the different columns. This was not allowed in [8]. Using the following definitions:

$$
\eta_{c}=\frac{c_{3}}{c_{1}}=\frac{c_{3}}{c_{2}} \quad \eta_{s}=\frac{s_{3}}{s_{1}}=\frac{s_{3}}{s_{2}},
$$

we have presented on Fig. 3, for $G=0$ and for different values of $J, \eta_{c}$ and $\eta_{s}$ as functions of the temperature. Because $G=0, \eta_{c}=\eta_{s}=\eta$ and the curves are identical for each $J$. This ratio has a well-defined value only below the critical temperature $T_{c}$. It is to be noticed that for all cases presented, $\eta$ is always larger than unity. For $J<0$, the behavior is even larger than for $J>0$. As a consequence, the displaced columns show a larger amplitude for the modulated phases. 


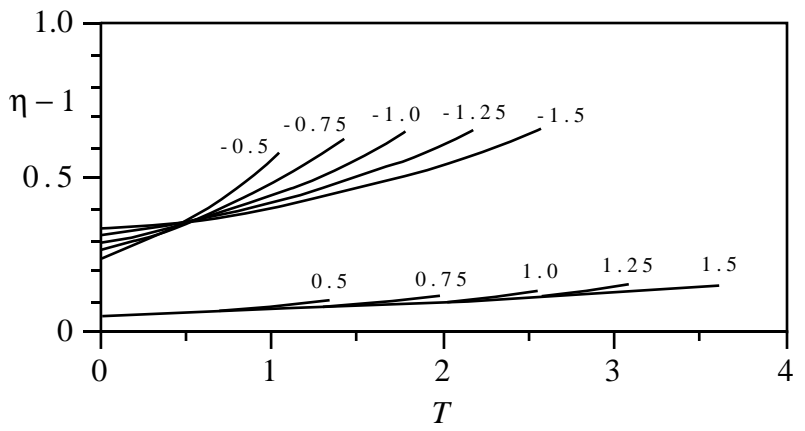

FIG. 3. $\eta(T)$ curves for $G=0$ and different values of $J$. $\eta(T)$ is defined only for $T$ below the critical temperature $T_{c}$, depending on the $J$ value.

For $G \neq 0$, the rotational invariance is broken and we expect non-circularly polarized phases. Numerically, we still found $\varphi_{\mu}^{s}-\varphi_{\mu}^{c}= \pm \frac{\pi}{2}$ and, if $|J| \geq|G|$, a $(+++)$ configuration is realized. If $|J|<|G|$, we have a $(++-)$ configuration, as previously found in [11] (see Fig. 4). The inter-column relative phases are related to the sign of $J+G$ for the cosines and of $J-G$ for the sines. For $J+G>0$,

$$
\varphi_{1}^{c}=\varphi_{2}^{c}=\varphi_{3}^{c},
$$

while for $J+G<0$,

$$
\varphi_{1}^{c}=\varphi_{2}^{c}=\varphi_{3}^{c} \pm \pi .
$$

For $J-G>0$,

$$
\varphi_{1}^{s}=\varphi_{2}^{s}=\varphi_{3}^{s},
$$

while for $J-G<0$,

$$
\varphi_{1}^{s}=\varphi_{2}^{s}=\varphi_{3}^{s} \pm \pi
$$

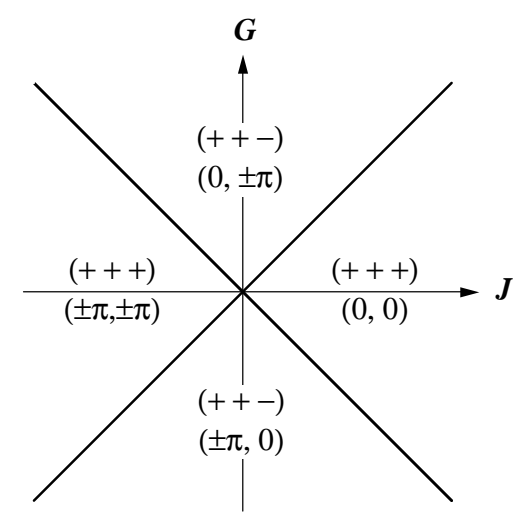

FIG. 4. Helicity patterns and phase differences in the $J-G$ plane. The first number $(0$ or $\pm \pi)$ is the difference $\varphi_{3}^{c}-\varphi_{1}^{c}$, and the second is $\varphi_{3}^{s}-\varphi_{1}^{s}$.
These phase relationships divide the $J-G$ plane in four quadrants, as seen in Fig. 1 . This diagram possesses an athermal character since these relative phases are the only variables having an influence on the sign of each term of $F, \cos \frac{1}{2} q$ being always positive. However, it is important to stress that the phases (and the phase relationships) have a physical meaning only when their corresponding amplitudes are nonzero. From these partial results, we may rewrite $F$ in a simpler form:

$$
\begin{aligned}
F_{T}=2 T & \left\{2 c_{1}^{2}+c_{3}^{2}+2 s_{1}^{2}+s_{3}^{2}\right. \\
+ & \left.\frac{1}{4}\left[3\left(2 c_{1}^{4}+c_{3}^{4}+2 s_{1}^{4}+s_{3}^{4}\right)+2\left(2 c_{1}^{2} s_{1}^{2}+c_{3}^{2} s_{3}^{2}\right)\right]\right\}, \\
F_{J}= & -\left(\cos q+J_{2} \cos 2 q\right)\left(2 c_{1}^{2}+c_{3}^{2}+2 s_{1}^{2}+s_{3}^{2}\right) \\
& -3(J+G) c_{1}^{2}-12|J+G|\left(\cos \frac{1}{2} q\right) c_{1} c_{3} \\
& -3(J-G) s_{1}^{2}-12|J-G|\left(\cos \frac{1}{2} q\right) s_{1} s_{3} .
\end{aligned}
$$

The absolute values of some couplings reflects the helicity choice made in order to minimize $F$. The positive signs of the coefficients of $c_{1}^{2} s_{1}^{2}$ and $c_{3}^{2} s_{3}^{2}$ favorize competition between $c$ and $s$ variables.

\section{Phase boundaries at $T_{c}$}

In this section, we investigate the first order-disorder transition to appear when lowering the temperature, that is, the highest temperature for which at least one order parameter is nonzero. This resulting critical temperature and the boundary between different phases should agree with the results found in Section A. This is presented in order to express the consistency of the Landau theory and its numerical treatment. For each parameter set $\left(T, J, G, J_{2}\right)$, we minimize $F$ defined with the expressions (32) and (33); using (22) and (23) would be equivalent, but numerically inefficient. The critical temperature is found by selecting $T$ such that one of $c_{1}, c_{3}, s_{1}$ or $s_{3}$ is as small as possible, but nonzero. We have recovered the diagram of Fig. 2: $T_{c}$ and $q_{c}$ being identical to those found in Section A. We also conclude that the transitions are always of second order, even with sixth-order terms in the expansion of $F_{T}$.

For $q_{c}=0$, that is, when $|J|$ or $|G|$ are large enough, some analytical results as easily obtainable. The matrix $\left\{H_{F}\right\}_{i j}=\left\{\partial^{2} F / \partial x_{i} \partial x_{j}\right\}$, where $\mathbf{x}$ is a vector constructed from the order parameters $\left(\mathbf{x}=\left(c_{1}, c_{3}, s_{1}, s_{3}\right)\right)$, defines the local convexity of $F$. We diagonalize $H_{F}$ to express this convexity along some eigendirections: $h_{F}$, the four eigenvalues of $H_{F}$, are the convexity coefficients. For each parameter set $\left(T, J, G, J_{2}\right), F$ is minimal for a particuliar $\mathbf{x}$. For the algebric form of the present $F$, the sign of each convexity coefficient at $\mathbf{x}=\mathbf{0}$ indicates whether $\mathbf{0}$ is a minimum or not: $F$ is minimal at $\mathbf{0}$ if every coefficient is positive, but one or more negative coefficients means that $\mathbf{0}$ is no longer a minimum. A vanishing eigenvalue of $H_{F}$ at $\mathbf{x}=\mathbf{0}$ corresponds to a second-order transition. 
$H_{F}$ has two distinct, doubly degenerate eigenvalues given by

$$
\begin{aligned}
h_{F}^{c, s}= & 3\left[2 T-\left(1+J_{2}\right)-(J \pm G)\right] \\
& +\sqrt{\left[2 T-\left(1+J_{2}\right)-3(J \pm G)\right]^{2}+144(J \pm G)^{2}} .
\end{aligned}
$$

$h_{F}^{c}$ correspond to $J+G$ and $h_{F}^{s}$ to $J-G$. For a set $\left(J, G, J_{2}\right), T$ is the critical temperature $T_{c}$ when every $h_{F}$ is positive at $\mathbf{x}=\mathbf{0}$, but at least one vanishes. The eigenvalues vanish at the following temperatures:

$$
T_{c}^{c, s}=\frac{1}{2}\left(1+J_{2}\right)+\frac{3}{8}(J \pm G)+\frac{3}{8}|J \pm G| \sqrt{33} .
$$

We kept the solution that keeps $T_{c}$ positive for any $J$ and $G$. These expressions coincide with (14) if $q_{c}=0$. The cosine or sine components order depending on which of the two temperatures $T_{c}^{c}$ or $T_{c}^{s}$ is maximum. We have a phase boundary between the two types of order for $T_{c}^{c}=T_{c}^{s}$. This equality is equivalent to (16). For $q_{c} \neq 0$, the same analysis leads easily to (14).

For a small $q_{c}$, we expand $T_{c}^{c, s}$ in powers of $q_{c}^{2}$. The $q_{c}^{4}$ coefficient is negative. The boundary between $q_{c}=0$ and $q_{c} \neq 0$ phases occurs when the $q_{c}^{2}$ coefficient vanishes. We have the relation

$$
|J \pm G|=\frac{22}{\sqrt{33}}\left(-J_{2}-\frac{1}{4}\right),
$$

which defines the boundaries on Fig. 2 (the dashed lines).

\section{Thermal phase diagrams}

Below the critical temperature, and for $J$ and $G$ exactly lying on the $|G|=-g\left(J, J_{2}\right) J$ curve, both the $c$ and $s$ components order. For the same $G$ and $J_{2}$ but for a higher $J$, the $c$ components order first, followed at lower temperature by the $s$ components, and conversely for a lower $J$. Consequently, the concomitant ordering of both the $c$ and $s$ components exists for a range of $J$. From the equivalence of the $\mu=1$ and $\mu=2$ columns, we have observed that $c_{1}=c_{2}$ and $s_{1}=s_{2}$. Conversely, but because of their mutual hindrance, $c_{1}<c_{3}$ and $s_{1}<s_{3}$.

We show in Fig. 5 the thermal phase diagrams for constant values of $G$ and $J_{2}=-1$. The symmetry of $F$ with respect to $G \leftrightarrow-G$ allows us to consider only $G \geq 0$. Each diagram has a high-temperature disordered phase (denoted $d$ ) and, at lower temperatures, ordered phases $\sin$ and $\cos$. For $q \neq 0$, these phases are denoted Msin and Mcos. Just below the $|G|=-g\left(J, J_{2}\right) J$ curve is a mixed $\sin +\cos$ phase that transforms continuously from sin to cos when approaching this region from large positive and negative $J$. For $q \neq 0$, the region $\sin +\cos$ is an elliptical phase denoted $E$.

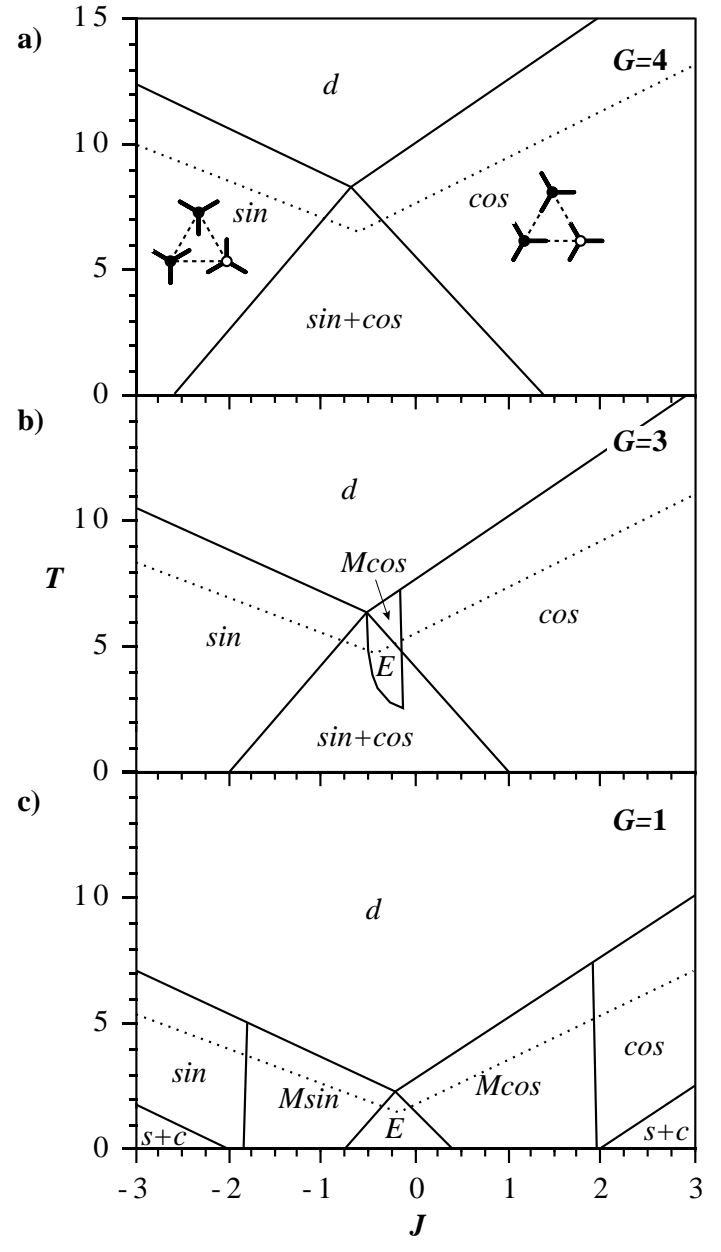

FIG. 5. Thermal phase diagrams for strong transverse couplings (see text). For $G=4$ (a) there is no modulated phases. For $G=3$ (b) the modulated phases are reentrant. The dotted lines indicate the limit of validity of the free energy limited power series expansion, below which at least one order parameter is greater than 0.5 . The insets schematically illustrate the orientations of the three molecules in a plaquette for sin and cos phases.

For high values of $|J|$ or $|G|, q$ remains zero below $T_{c}$, as shown in Fig. 5a. For small $|G|$ and increasing $|J|$, steep boundaries between modulated and non-modulated phases are crossed (see Fig. 国): $q$ vanishes continuously from Mcos to cos and from Msin to sin. Inversely, for small $|J|$ and increasing $|G|$, a reentrant boundary is crossed: a modulated elliptical phase reappears with increasing temperatures (see Fig. 5b). For high values of $|J|$, below the critical temperature, the disordered variables ( $c$ in the $\sin$ phase and $s$ in the cos phase) order at lower temperatures, forming $\sin +\cos$ phases. For $G=1$ (Fig. Fic), we see the beginning of this phase for $|J|>\sim 2$. The phase boundaries are parallel to the sin and $\cos$ boundaries with the disordered phase. These low temperature phases are not shown on Figs $5 \mathrm{a}$ and $5 \mathrm{~b}$, but 
they occur respectively for $|J|>\sim 6$ and 8 . Because helicity configurations have meaning only when $c_{\mu}, s_{\mu}$ and $q$ are nonzero, that is, for $E$ phases, the diagrams of Fig. 5 do not show the richness of Fig. 1 . For the choice of parameters, the central $E$ phases in Fig. 5 are $(++-)$ phases. For smaller values of $|G|$, it is expected that a phase boundary between $E$ phases having $(++-)$ and $(+++)$ configurations would be observable.

Indeed, the quasi one-dimensional regime, where $\left|J_{1}\right|,\left|J_{2}\right| \gg|J|,|G|$, is interesting because it is more realistic for HHTT, with $E$ phases having different helicity patterns. We show in Fig. 6 thermal phase diagrams for $G$ from 0.1 to 0.5 . Both the $E(+++)$ and $E(++-)$ phases exist and are sometimes adjacent. On the $J$ interval presented in Fig. 6, all the ordered phases are modulated. The temperature "depth" of the Msin and Mcos phases is approximately proportional to $|G|$ and independent of $J$.

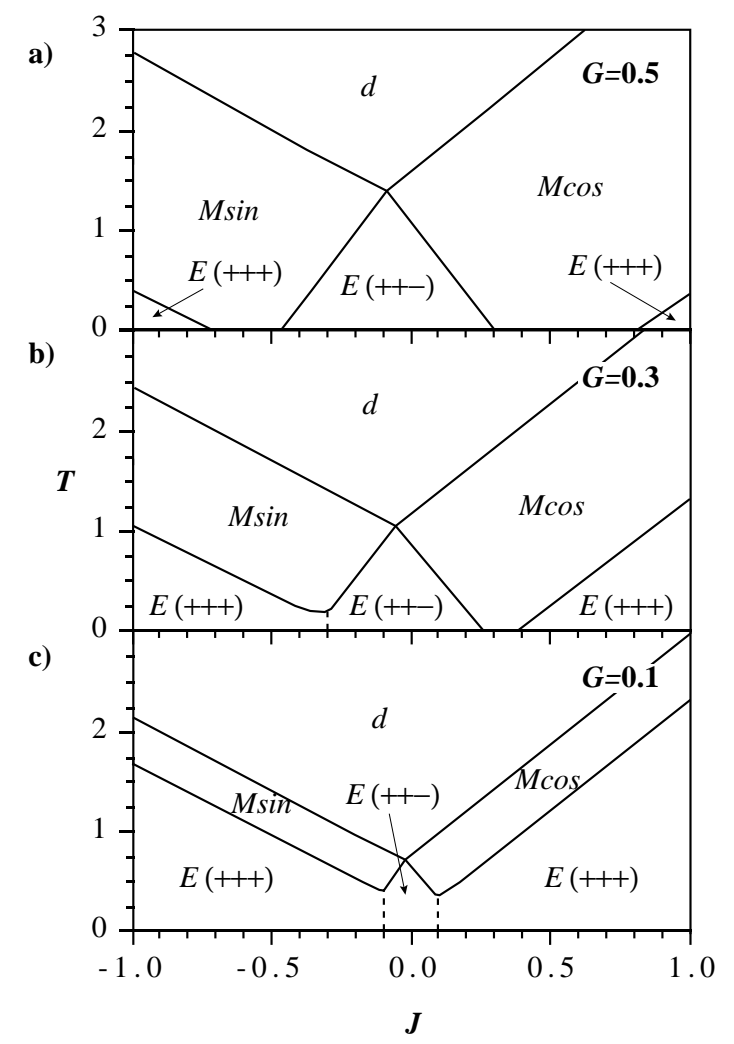

FIG. 6. Thermal phase diagrams in the quasi-onedimensional regime (see text). For $G=0.5$ (a) the $E(++-)$ and $E(+++)$ are separated. For $G=0.3$ (b) and $G=0.1$ (c), dashed lines denote first-order helicity reversal transitions.

Fig. 7illustrates schematically the positions and orientations of the molecules for different phases encountered in the diagrams of Fig. 6, in the case where $G>0$. Some are also present in Fig. 5 . The length of the tails represent the amplitude of ordering. The Msin phase (Fig. Ta) shows modulated order with uniform orientations along the columns and where column 3 is in phase opposition. For $G>0$, the Mcos phase (Fig. $7 \mathrm{~b}$ ) is a modulated ferromagnetic-like state. The maximum lengths of the tails shows that column 3 is more ordered than columns 1 and 2. The corresponding sin and cos phases are illustrated in Fig. Fa. Fig. Gc to d represent the various elliptical phases $E$ encountered from left to right in Fig. 6.
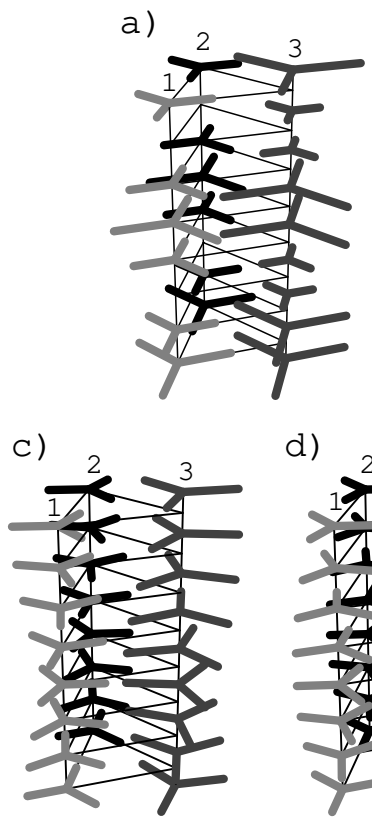

d)
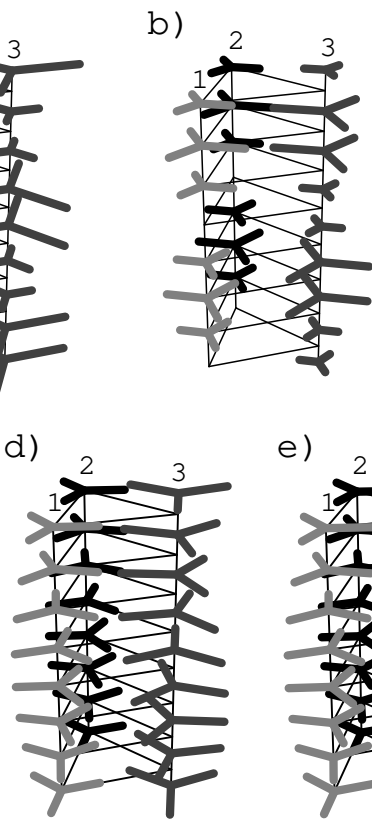

e)

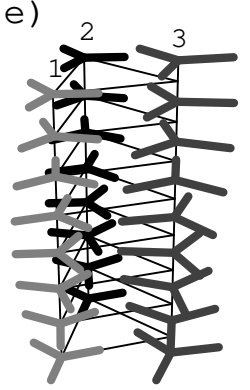

FIG. 7. Schematic illustration of the orientations of molecules and amplitudes of mean values with $G>0$ for a) the Msin phase with column 3 in phase opposition, b) the Mcos phase with column 3 in phase conjunction, c) the $E(+++)(\pi, \pi)$ phase, d) the $E(++-)(0, \pi)$ phase and e) the $E(+++)(0,0)$ phase.

\section{MONTE CARLO SIMULATIONS}

\section{A. Method and algorithm}

We use the Metropolis algorithm (see 18 on general Monte Carlo methods) to simulate a three-dimensional lattice of XY variables. Our goal is to obtain the essential features of the thermal phase diagram of the system (4), restricting ourselves to the quasi one-dimensional regime.

The above method has been implemented in conjunction with the "spiraling" algorithm [19,20], which simulates incommensurate helical phases by relaxing the constraint associated with the finite size of the lattice. Outside the critical regime, the results are in principle independent of the lattice size. This method is applied on each column of $N$ XY variables $\left\{\phi_{1}, \ldots, \phi_{N}\right\}$. We simulate the neighbors of $\phi_{1}$ and $\phi_{2}$ with phantom sites (denoted by a prime) related to sites of the opposite extremity of the column: 


$$
\phi_{N-1}^{\prime}=\phi_{N-1}-N \Delta, \quad \phi_{N}^{\prime}=\phi_{N}-N \Delta .
$$

For the sites $\phi_{N-1}$ and $\phi_{N}$, we use the phantom neighbors

$$
\phi_{1}^{\prime}=\phi_{1}+N \Delta, \quad \phi_{2}^{\prime}=\phi_{2}+N \Delta .
$$

The reader will note that, for $\Delta=0$, simple periodic boundary conditions are recovered. $\Delta$ is an effective field representing an additional indefinite length of a lattice modulated with a constant pitch. For the system to select its own boundary conditions, we consider $\Delta$ as a thermodynamic variable. Each Monte Carlo step of the spiraling algorithm consists in $N$ ordinary trial flips of the $\phi$ variables and a single trial flip of $\Delta$. We modify $\Delta$ by a small random angle $\delta \Delta$. In order for the new $\Delta$ to be compatible with the above equations, a twist must be imposed on the lattice:

$$
\phi_{i} \leftarrow \phi_{i}+(i-1) \delta \Delta .
$$

The new state has a new total energy, and is then tested for acceptance with the Metropolis algorithm. We may compare the spiraling algorithm to a high-order meanfield approximation, where the exact clusters have the size of the finite lattice used in the simulation. Close to a transition, when the correlation length is large relative to the cluster size, this approximation loses its validity

We simulate a $6 \times 6$ triangular lattice of columns, that is, 12 groups of three columns. We use periodic boundary conditions in the plane and the spiraling algorithm in the columns' direction. Each column has its own $\Delta$ variable.

For two neighboring columns with non-planar interactions $\left(J^{\prime}\right.$ and $\left.G^{\prime}\right)$, the spiraling algorithm introduces some small energy discrepancies. This problem arises from the non-equivalence of some of the couplings at the edges of the lattice. Due to the relatively small energy involved in this boundary effect (especially for a quasi one-dimensional system), it was neglected.

We identify the different phases with the nonzero values of the Fourier coefficients of the $x$ and $y$ components defined by:

$$
\begin{aligned}
& a_{\mu}^{c}(\tilde{q})=\frac{2}{12 N} \sum_{(i, j) \in R_{\mu}} \sum_{k} \cos \phi_{i j k} \cos \tilde{q} z_{k}, \\
& b_{\mu}^{c}(\tilde{q})=\frac{2}{12 N} \sum_{(i, j) \in R_{\mu}} \sum_{k} \cos \phi_{i j k} \sin \tilde{q} z_{k} \\
& a_{\mu}^{s}(\tilde{q})=\frac{2}{12 N} \sum_{(i, j) \in R_{\mu}} \sum_{k} \sin \phi_{i j k} \cos \tilde{q} z_{k}, \\
& b_{\mu}^{s}(\tilde{q})=\frac{2}{12 N} \sum_{(i, j) \in R_{\mu}} \sum_{k} \sin \phi_{i j k} \sin \tilde{q} z_{k}
\end{aligned}
$$

with $z_{k}=k+\frac{1}{2} \delta_{\mu 3} . \quad R_{\mu}$ represents the sublattice of $\mu$ columns and $N$ is the number of sites in each column.
Using these coefficients, we construct the order parameters:

$$
\begin{aligned}
& c_{\mu}(\tilde{q})=\sqrt{a_{\mu}^{c}(\tilde{q})^{2}+b_{\mu}^{c}(\tilde{q})^{2}}, \\
& s_{\mu}(\tilde{q})=\sqrt{a_{\mu}^{s}(\tilde{q})^{2}+b_{\mu}^{s}(\tilde{q})^{2}} .
\end{aligned}
$$

The $\left\langle c_{\mu}(\tilde{q})\right\rangle$ and $\left\langle s_{\mu}(\tilde{q})\right\rangle$ profiles show a maximum at $\tilde{q}=q_{0}$, and may show secondary peaks. Indeed, it has been shown 21 that a third harmonic at $q_{3}=3 q_{0}$ should appear for linearly polarized columns. In our simulations, this third harmonic should be an indication of such a linear polarization. We also construct the relative angular phases of the columns defined by:

$$
\begin{aligned}
& \cos \left(\phi_{\mu}^{s}-\phi_{\mu}^{c}\right)(\tilde{q})=\frac{a_{\mu}^{s}(\tilde{q}) a_{\mu}^{c}(\tilde{q})+b_{\mu}^{s}(\tilde{q}) b_{\mu}^{c}(\tilde{q})}{s_{\mu}(\tilde{q}) c_{\mu}(\tilde{q})}, \\
& \sin \left(\phi_{\mu}^{s}-\phi_{\mu}^{c}\right)(\tilde{q})=\frac{b_{\mu}^{s}(\tilde{q}) a_{\mu}^{c}(\tilde{q})-a_{\mu}^{s}(\tilde{q}) b_{\mu}^{c}(\tilde{q})}{s_{\mu}(\tilde{q}) c_{\mu}(\tilde{q})}, \\
& \cos \left(\phi_{\mu+1}^{c}-\phi_{\mu}^{c}\right)(\tilde{q})=\frac{a_{\mu+1}^{c}(\tilde{q}) a_{\mu}^{c}(\tilde{q})+b_{\mu+1}^{c}(\tilde{q}) b_{\mu}^{c}(\tilde{q})}{c_{\mu+1}(\tilde{q}) c_{\mu}(\tilde{q})}, \\
& \sin \left(\phi_{\mu+1}^{c}-\phi_{\mu}^{c}\right)(\tilde{q})=\frac{b_{\mu+1}^{c}(\tilde{q}) a_{\mu}^{c}(\tilde{q})-a_{\mu+1}^{c}(\tilde{q}) b_{\mu}^{c}(\tilde{q})}{c_{\mu+1}(\tilde{q}) c_{\mu}(\tilde{q})}, \\
& \cos \left(\phi_{\mu+1}^{s}-\phi_{\mu}^{s}\right)(\tilde{q})=\frac{a_{\mu+1}^{s}(\tilde{q}) a_{\mu}^{s}(\tilde{q})+b_{\mu+1}^{s}(\tilde{q}) b_{\mu}^{s}(\tilde{q})}{s_{\mu+1}(\tilde{q}) s_{\mu}(\tilde{q})},
\end{aligned}
$$

$$
\sin \left(\phi_{\mu+1}^{s}-\phi_{\mu}^{s}\right)(\tilde{q})=\frac{b_{\mu+1}^{s}(\tilde{q}) a_{\mu}^{s}(\tilde{q})-a_{\mu+1}^{s}(\tilde{q}) b_{\mu}^{s}(\tilde{q})}{s_{\mu+1}(\tilde{q}) s_{\mu}(\tilde{q})} .
$$

We compare $\left\langle\cos \left(\phi_{\mu}^{s}-\phi_{\mu}^{c}\right)\left(q_{0}\right)\right\rangle$ to the mean-field expression $\cos \left(\phi_{\mu}^{s}-\phi_{\mu}^{c}\right)$, and so on for each mean value.

The amplitudes (41) converge relatively fast, but the angular phases (42) converge much more slowly: they are ratios of fluctuating quantities. For some simulations, we are only interested in the $\left\langle c_{\mu}(\tilde{q})\right\rangle$ and $\left\langle s_{\mu}(\tilde{q})\right\rangle$ values, and we have simulated only $2000 \mathrm{MCS} / \mathrm{S}$, including $1000 \mathrm{MCS} / \mathrm{S}$ for thermalization. $N$, the number of sites per column, was taken to be 40 . However, many more steps was needed to obtain the relative angular phases (25000 MCS/S, including $5000 \mathrm{MCS} / \mathrm{S}$ to thermalize). In these cases, $N$ was taken to be 12 . To improve numerical efficiency, we discretize the $\phi$ and $\Delta$ variables into 256 values from 0 to $2 \pi$. 
All simulations are done with $G=0.1$ and $J_{2}=-1$, a quasi one-dimensional limit. We perform the simulations by gradually decreasing the temperature for each value of $J$. This allows a greater numerical stability for lowtemperature phases and retains the helicity sign $((++\mp)$ or $(-- \pm))$ for all temperatures.

Preliminary simulations on a single plaquette of three columns were done to compare the spiraling algorithm with periodic boundaries conditions in some reasonable computing time. For $N=40$, simulations using periodic boundaries along columns give very similar results compared to simulations with the spiraling algorithm, except for smaller mean values (because of higher fluctuations) and slightly displaced peaks. However, the difference is pronounced for $N=12$, where the spiraling algorithm broadens the principal peak, while periodic boundaries conditions destroy the whole spectrum.
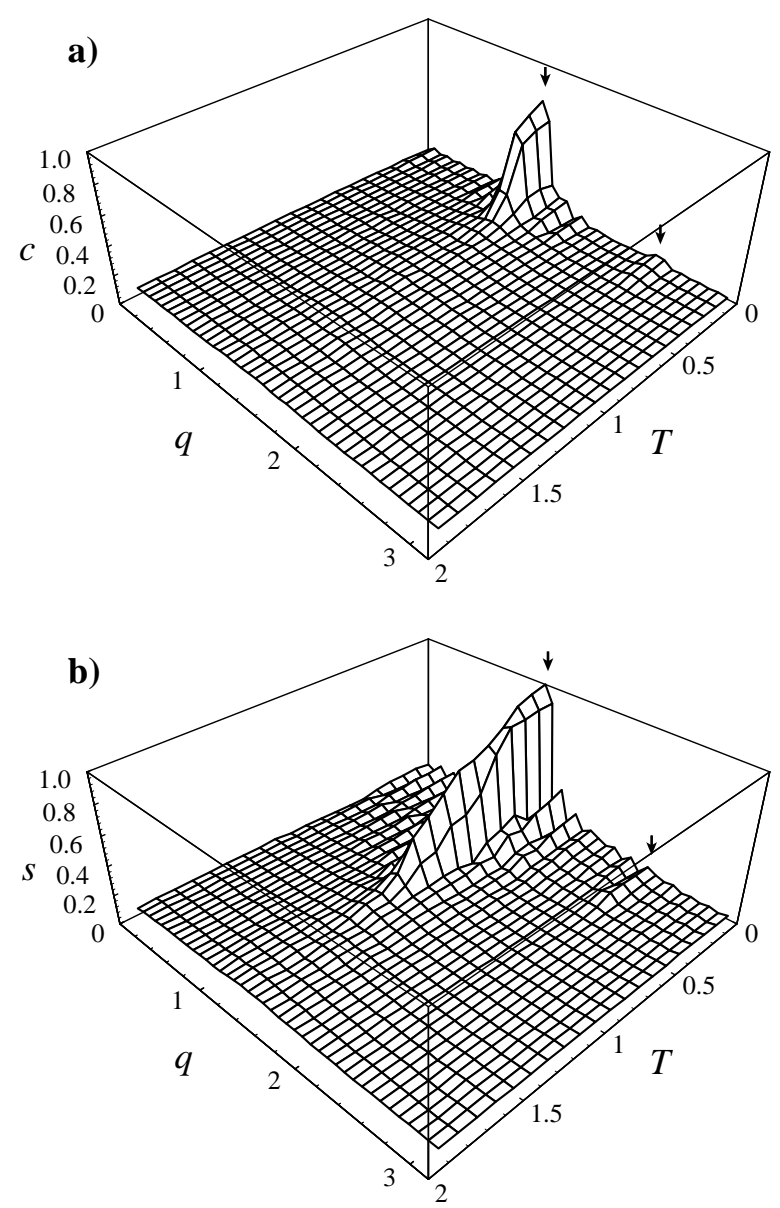

FIG. 8. a) $\left\langle c_{1}(q)\right\rangle(T)$ and b) $\left\langle s_{1}(q)\right\rangle(T)$ profiles for $J=-0.15, G=0.1$ and $J_{2}=-1$. The arrows indicate the first and third harmonics. The $q_{0}$ peaks appear at $T \approx 0.4$ for $c_{1}$ and $T \approx 0.9$ for $s_{1}$.

\section{B. Thermal phase diagrams}

Fig. 8 shows a typical result for the order parameters, from which the thermal phase diagram is constructed: the profiles $\left\langle c_{1}(\tilde{q})\right\rangle(T)$ and $\left\langle s_{1}(\tilde{q})\right\rangle(T)$. Notice that both $q_{0}$ and $q_{3}$ peaks appear at specific temperatures $\left(q_{3}\right.$ is folded in the $[0, \pi]$ interval). The $\left\langle c_{\mu}(\tilde{q})\right\rangle$ and $\left\langle s_{\mu}(\tilde{q})\right\rangle$ profiles show that, for $J=-0.15$, a decreasing temperature drives the system from $d$ phase, to Msin phase and finally to $E$ phase. The critical temperatures are arbitrarily taken to be the points at which the amplitude of the peak is half its maximum value.

The thermal phase diagram is constructed by repeating the simulations for many $J$ values. Fig. 9 shows the diagram for $G=0.1$ and $J_{2}=-1$. We always obtain $\left\langle\cos \left(\phi_{\mu}^{s}-\phi_{\mu}^{c}\right)\left(q_{0}\right)\right\rangle \approx 0$ and $\left\langle\sin \left(\phi_{\mu}^{s}-\phi_{\mu}^{c}\right)\left(q_{0}\right)\right\rangle \approx \pm 1$, with signs corresponding to the mean-field helicity configurations: $(++-)$ or $(--+)$ for $|J|<0.1$ and $(+++)$ or $(---)$ for $|J|>0.1$. Some longer simulations were done to establish clearly the phase differences, at $J=-0.15$, $-0.05,0.05$ and 0.15. Given some high-enough $\left\langle c_{\mu}\left(q_{0}\right)\right\rangle$ and $\left\langle s_{\mu}\left(q_{0}\right)\right\rangle$ amplitudes, every relative phase is compatible with the mean-field calculations and the results just cited. Moreover, many short simulations where done on a single plaquette of three columns $(N=40)$ and, although no phase transition is observed (the system is onedimensional), the phase differences are in excellent agreement with Fig. 4 .

Contrarily to the mean-field results (Fig. 6.c), the $E$ phases with different helicity configurations were not found to be adjacent: a linearly polarized phase opens up between the $E(+++)$ and $E(++-)$ phases. On the diagram, for a given value of $J$, a dot at $T=0$ means that no phase transition was clearly identified when lowering the temperature.

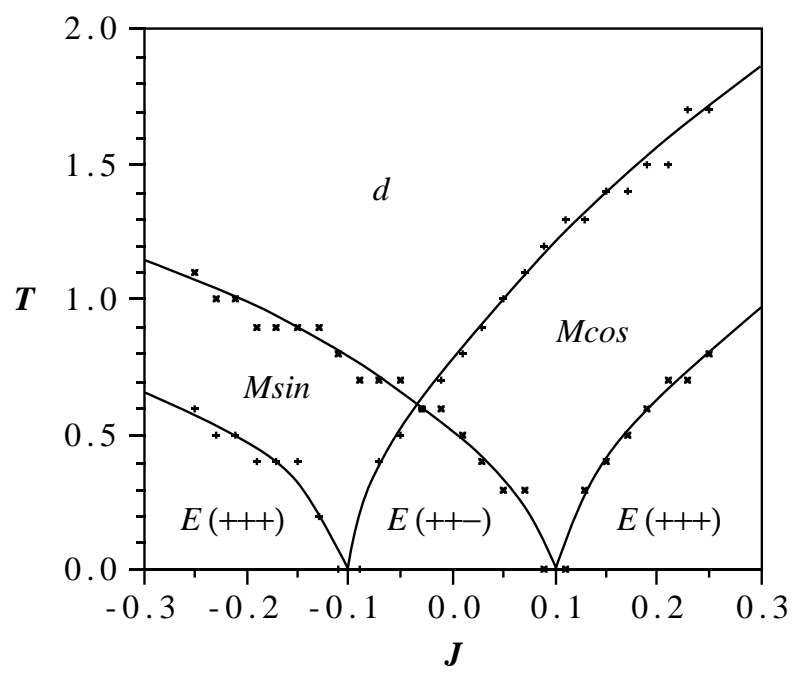


FIG. 9. Thermal phase diagram for $G=0.1$ and $J_{2}=-1$ from Monte Carlo simulations. The continuous line is a guide to the eye (see Fig. 6ic for the mean-field diagram).

\section{DISCUSSION AND CONCLUSION}

X-ray measurements on HHTT have lead to the observation of a sequence of phases as temperature is lowered. Two neighboring phases $\left(D_{h d}\right.$ and $H$ ) at intermediate temperatures have a triangular structure of columns of molecules. Entering the $H$ phase from the $D_{h d}$ phase involves a mutual and concomitant ordering of both the position and orientation of the molecules along the columns. However, it is immediately realized that the positions of the molecules are rapidly frozen as compared to their orientations, being submitted to more stringent intermolecular forces. This is the main justification for the model studied in this paper, limited to orientational degrees of freedom. The obtained results and in particular the general trend of the thermal phase diagram should be useful to understand the behavior of HHTT inside the $H$ phase.

The first result of interest is the ordering of linearly polarized phases at $T_{c}$ with a finite wavenumber for the amplitude modulation in the columnar direction, whose value is decreasing with increasing values of the intermolecular couplings and eventually vanishes. A similar disappearance of the amplitude modulation has been predicted [8] at $T=0$ where it then shows up as an unwinding of the helical pitch. At $T_{c}$, for large $|J|$ and $|G|$, a boundary at a constant slope of magnitude $\sqrt{33}$ is predicted between two linearly polarized phases. It is to be noticed that this behavior may be seen as a reminiscent effect of the two boundary structures predicted at $T=0$ between linearly polarized phases. At $T=0$, the constant slopes of the boundaries are respectively -3 and -5 . As seen on Fig. 5a, this $T=0$ behavior is perfectly predictible at large $G$.

The frustration between ordered columns of molecules with an octupolar moment on a triangular lattice is very high. Part of this frustration is relaxed by freezing the positions of the molecules and displacing one of every three columns by half a lattice spacing in the columnar direction. However, for negative value of the interaction parameter $J$, even under these conditions, substantial orientational frustration remains. At $T=0$, with only constant amplitude phases, a non-colinear distorted $120^{\circ}$ phase was obtained with global relative angular phases between the columns which depended on the pitch of the helical modulation of the columns. In the present case, allowing for different amplitudes of the modulation for the displaced columns compared to the undisplaced columns, the resulting configuration is colinear and the relative angular phases is independent of the pitch. This angular configuration, typical of unfrustrated systems, is only achieved through a larger amplitude of modulation for the displaced columns as compared to the undisplaced ones, as shown if Fig. 3 .
For moderate values of $G$ as compared to the intracolumnar couplings (Fig. 6), when there exists elliptical phases $E$, both the helicity patterns $(+++)$ and $(++-)$ are predicted. Also it is to be noted that the temperature range over which the linearly polarized and modulated phases exist, narrows down with decreasing value of $G$. Under these conditions, it is expected that we rapidly enter the elliptical phases on lowering the temperature below $T_{c}$. In this temperature range, where our model, which freezes the positional degrees of freedom rapidly under $T_{c}$, is certainly valid, and for $|G|>|J|$, the elliptical phase $(++-)$ is predicted. Recall that the $(++-)$ phase is the one observed [4] for HHTT in the $H$ phase. We then conclude that HHTT is a quasi one-dimensional system where the molecular orientations are dominated by the non-rotationally invariant interaction between octupolar moments located on a distorted triangular lattice.

The finite temperature Monte Carlo simulations on finite size systems has confirmed remarkably the mean field results. The one important difference is that the $(++-)$ and $(+++)$ configurations are not seen to be adjacent in Monte Carlo simulations where they were predicted to have a common boundary in the mean field approximation. The hard-spin constraint $\left|\mathbf{S}_{\mu m}\right|=1$ is automatically satisfied in the Monte Carlo simulations. At low temperature and in the mixed regime $|J| \approx|G|$, the system is indecisive between configurations where all the columns have identical helicities and opposite helicities between the displaced columns and the undisplaced columns. Based on the hard-spin contributions which are optimal for non-helical phases, the system seems to be driven to zero temperature while retaining either a modulated cos or sin phase according to the sign of $J$. This result probably manifest itself into the rare occurence of helical phases experimentally.

Regarding the $D_{h d} \rightarrow H$ transition in HHTT, this work raises the question of the detailed nature of the observed $H$ phase near the transition: is there a linearly polarized " $H$ phase"? The presence of a linearly polarized modulated phase would be an indication of the chiral octupolar nature of the molecule (the $G$ coupling). Such phases could be observed as a third harmonic in the modulation of the columns in X-ray measurements. The most important result of this paper is the higher amplitude predicted for the displaced columns as compared to the undisplaced columns. This predicted behavior would affect the $\mathrm{X}$-rays results and in particular the relative amplitude and thermal broadening of the Bragg's peaks. The $J$ and $G$ values may be varied by using discoid compounds other than HHTT or by the application of surface pression. 


\section{ACKNOWLEDGMENTS}

The authors are grateful to M.L. Plumer for many discussions. G.L. would like to thank W.M. Saslow for helpful suggestions related to the simulations. This work is supported by NSERC (Canada) and by FCAR (Québec).

[1] S. Chandrasekhar, Liquids crystals (2nd edition), Cambridge University Press (1992).

[2] P.-G. de Gennes and J. Prost, The physics of liquid crystals (2nd edition), Oxford University Press (1993).

[3] E. F. Gramsbergen, H. J. Hoving, W. H. de Jeu, K. Praefke and B. Kohne, Liq. Cryst. 1, 397 (1986).

[4] E. Fontes, P. A. Heiney and W. H. de Jeu, Phys. Rev. Lett. 61, 1202 (1988).

[5] E. Fontes, Ph.D. dissertation, University of Pennsylvania (1989).

[6] S. Idziak, Ph.D. dissertation, University of Pennsylvania (1992).

[7] A. Caillé and M. Hébert, Phys. Rev. E 54, R4544 (1996).

[8] M. Hébert and A. Caillé, Phys. Rev. E 53, 1714 (1996).

[9] M. Hébert and A. Caillé, Phys. Rev. E 51, R1651 (1995).

[10] M. Hébert and M. L. Plumer, Phys. Rev. E 54, 550 (1996).

[11] M. L. Plumer, A. Caillé and O. Heinonen, Phys. Rev. B 47, 8479 (1993).

[12] M. Pesquer, M. Cotrait, P. Marsau, V. Volpilhac, J. Phys. France 41, 1039 (1980).

[13] M. Cotrait, P. Marsau, M. Pesquer, V. Volpilhac, J. Phys. France 43, 355 (1982).

[14] W. Selke, Spatially Modulated Structures in Systems with Competing Interactions, in Phase Transitions and Critical Phenomena, vol. 15, Academic Press (1992).

[15] S. Redner and H. E. Stanley, Phys. Rev. B 16, 4901 (1977).

[16] H.-Y. Choi, A. B. Harris and E. J. Mele, Phys. Rev. B 40, 3766 (1989).

[17] P. Bak and J. von Boehm, Phys. Rev. B 21, 5207 (1980).

[18] K. Binder (editor), Monte Carlo Methods in Statistical Physics, Topics in Current Physics 7 (1979).

[19] W. M. Saslow, M. Gabay and W.-M. Zhang, Phys. Rev. Lett. 68, 3627 (1992).

[20] M. Collins and W. M. Saslow, Phys. Rev. B 53, 8533 (1996).

[21] M. B. Walker, Phys. Rev. B 22, 1338 (1980). 